\title{
Assessing Cancer Drugs for Reimbursement: Methodology, Relationship between Effect Size and Medical Need
}

\author{
Rima de Sahb-Berkovitch ${ }^{1}$, Marie-Christine Woronoff-Lemsi ${ }^{2}$, Mathieu Molimard ${ }^{3}$ and participants of Round \\ Table $n^{\circ} 7$ of Giens 2009*
}

1 Laboratoire Roche, Neuilly sur Seine, France

2 Centre Hospitalier Universitaire de Besançon, Besançon, France

3 Centre Hospitalier Universitaire de Bordeaux, Bordeaux, France

Text received 10 february 2010; accepted 26 avril 2010

\section{Keywords:} reimbursement; cancer drugs; overall survival; progression free survival; criteria;

Transparency Commission

\begin{abstract}
Reimbursement is assessed by the Transparency Commission from the Health Authority (HAS) using a medical benefit (SMR) score that gives access to reimbursement, an "improvement of medical service rendered" (ASMR) that determines the added therapeutic value, and the target population. Assessing cancer drugs for reimbursement raises the same issues as other therapeutic classes, with some key differences. Overall survival (OS) is considered by the Transparency Commission as the endpoint for assessing clinical benefit, and yet it is not an applicable primary endpoint in all types of cancer. Later lines of treatment, particularly during the development process, may make it difficult to interpret OS as the primary endpoint. Therefore, progression-free survival (PFS) for metastatic situations and disease-free survival (DFS) in adjuvant situations are wholly relevant endpoints for decisions on the reimbursement of a new cancer drug. Effect size is assessed using actuarial survival curves of the product versus the comparator, and it is difficult to summarise them into one single parameter. Results are generally interpreted based on median survival, which is fragmented because it only measures one point of the curve. The hazard ratio measures the effect of treatment throughout the duration of survival and is therefore more comprehensive in quantifying clinical benefit. Determining an effect size threshold for granting reimbursement is difficult given the diversity of cancer settings and the level of medical need, which influences assessment of the clinical relevance of the observed difference. Rapid progress in comparators (700 molecules in development) and the identification of predictive factors of efficacy (biomarkers, histology, etc.) during development may lead to different ASMR scores per population, or to the restriction of the target population to a subgroup of the marketing authorisation (MA) population in which the expected effect size is greater. To address these issues, the roundtable recommends the possibility of early scientific opinions by the office of the Transparency Commission in order to discuss comparators and the relevance of responder subgroups. It also recommends the possibility of granting a temporary ASMR, on condition of subsequent confirmation by production of data, when reimbursement appears justified in a subpopulation of the MA for which only subgroup analysis is available.
\end{abstract}

\section{Introduction}

The case for reimbursement is assessed by the Transparency Commission from the Health Authority (HAS) using a medical benefit (SMR) score that gives access to reimbursement. The SMR takes into account the seriousness of the treated condition (morbidity and mortality), the efficacy/safety ratio, the position of the drug in the therapeutic strategy (particularly in comparison to other available therapies) and the public health interest (disease burden, coverage of medical need and response versus need, ex-

\footnotetext{
* For a list of participants, see end of article.
}

trapolation of data into real life and impact on the healthcare system).

The SMR is termed major, or significant, moderate or low, giving access to reimbursement with scores ranging from $75 \%$ to $15 \%$, or insufficient to justify reimbursement.

The Commission also evaluates improvement in medical benefit (ASMR), which is added value relative to effect size, with regard to a comparator(s) or, failing which, therapeutic strategy. The assessment endpoints and methodological rules define a level of evidence and effect size is assessed based on clinical relevance and not merely statistical significance. Finally, the Commission 
defines the target population for which the opinion recommends reimbursement. This population may correspond to a subgroup of the population in the indication granted in the marketing authorisation MA).

The ASMR score does not directly affect reimbursement but rather the price level assigned by the Economic Committee on Health Products (CEPS).

The issue raised by assessing cancer drugs for reimbursement is related to the assessment endpoints. Overall survival (OS), which is considered by the Transparency Commission as the endpoint for assessing clinical benefit, is not applicable as the primary endpoint in all types of cancer, particularly slowly progressive diseases and when salvage treatments are available. Later lines of treatment, particularly during the development process, may make it difficult to interpret OS as the primary endpoint and it is not certain that the desired clinical benefit can always be measured in terms of survival as some cancers, due to improvements in management, become chronic diseases.

In addition to overall survival, what endpoints are therefore to be considered as measurements of clinical benefit in oncology and, irrespective of the endpoint used, what effect size is to be considered clinically significant to lead to reimbursement? Other questions surround the issue, such as consideration of medical need in the assessment of effect size, relevant comparators to be used in light of changing therapeutic strategies, and the target population.

\section{Methodology}

To answer the question, the Round Table first sought to review the efficacy endpoints currently taken into account in oncology, according to available European Medicine Agency (EMA) and Food and Drug Administration (FDA) guidelines. ${ }^{[1,2]}$

The literature was also reviewed, particularly analysis of the variability and validity of the endpoints in different types of cancer. Lastly, the opinions issued by the Transparency Commission on cancer drugs between early 2007 and June 2009 were analysed in an attempt to identify a rationale for selecting endpoints and a methodological approach to estimating effect size.

Secondly, cross-comparison tables for each efficacy endpoint were created on the basis of the research in order to rank their relevance for each type of cancer. Non-efficacy endpoints were also reviewed in order to determine the relevance of taking them into account for reimbursement. In this context, the question of data availability at the time of assessment for reimbursement was considered in order to define the documentation to be provided in supplemental post-registration studies.

\section{Relevant endpoints for the reimbursement of cancer drugs}

\section{1. Efficacy endpoints}

In oncology, overall survival (OS) is the endpoint with maximum clinical relevance because it is the primary clinical benefit sought for patients and a measurement without assessment bias: it is THE measurement of efficacy recognised by the Transparency Commission. It is defined as the time between randomisation and death by any cause, or the date of last information. The pitfall lies in the necessity of prolonged follow-up and enrolment of a large number of patients in certain cancers in which death is rare. In case of prolonged survival, non-cancer-related deaths often dilute the effect, increasing the difficulty of assessing the effect of treatment in the context of competitive risks from other causes of death including age. Lastly, for first-line treatments, the availability of subsequent lines of treatment, crossovers and switches are also factors complicating the interpretation of overall survival. ${ }^{[3-5]}$

Several other efficacy endpoints are assessed in trials, and guidelines are available for describing and assessing the antitumour activity of a treatment. ${ }^{[6,7]}$

Disease-Free Survival (DFS) in adjuvant situations and Progression-Free Survival (PFS) for metastatic situations are considered replacement endpoints for OS in certain cancers in which a correlation has been validated (colon cancer). ${ }^{[8-11]}$ In nonvalidated cases such as breast cancer, PFS is considered an intermediate endpoint. ${ }^{[12,13]}$ They are defined as the time between randomisation and local or distant recurrence for DFS, or the time between randomisation and progression, a second cancer or death by any cause for PFS. These endpoints have an undeniable clinical relevance because they measure the time during which there is no clinical evidence of tumour progression, thereby reflecting the anti-tumour action more directly than survival, and are not affected by later lines of treatment. The pitfall of these endpoints lies in the bias of the time of measurement since progression is usually assessed at the time specified by the protocol and not at the time of progression itself. Progress in knowledge and technology is changing the method of measuring progression and depriving assessors of an historical reference value. Finally, in terms of biometrics, a second reading by an independent review board or a centralised reading improve the specificity, sensitivity and reproducibility of the measurement without removing the biases. ${ }^{[14-17]}$

Tumour or biological response defined by the rate of response is an explanatory endpoint documenting the direct action of treatments on tumour mass. It involves the objective response rate (ORR) and the complete response rate, which are usually considered intermediate endpoints, except in malignant blood diseases, for which they are considered replacement endpoints. A good tool 
in the usual clinical follow-up of patients, they can be assessed early with regard to survival or progression and are useful in early development phases or in the absence of randomisation.

\subsection{Other endpoints}

Safety is taken into account by the Transparency Commission in order to counterbalance the assessment of efficacy and thereby judge therapeutic value. When the effect size is small, the frequency and intensity of adverse effects are considered in order to qualify the quality of the gain in survival. The Commission often bemoans the lack of quality-of-life data: tools are available in oncology but are difficult to use repeatedly in clinical trials and vary inter-individually, which reduces their relevance for deciding between treatments or therapeutic strategies.

As cancers become chronic, other tools such as examining patient preference or utility could be taken into account by the Transparency Commission in oncology.

\section{Assessment of effect size}

The notion of effect size for SMR and ASMR incorporates the effect (on what and which endpoint) and the size (how many). The assessment differs from MAs as the Commission particularly researches the clinical relevance of a statistically significant effect size by taking into account medical need, disease burden, target population and above all the capacity to extrapolate the results of trials to real-life practice (extrapolation). The comparative nature of data is also a key factor in assessing effect size.

\subsection{Median survival}

The primary endpoint, whether OS, PFS or DFS, is expressed in the form of survival curves. Assessment of effect size by the Commission is usually based on median survival, which is the assessment of survival at a time $t$. This expression of effect size is an easily understood endpoint but only represents a single point of the survival curve and in certain cases may not be reached in an arm at the time of evaluation.

\subsection{Hazard ratio}

The other expression of effect size is hazard ratio. It is the ratio of immediate risks of events between arm A (experimental arm) and arm B (control arm).

$$
H R=\frac{\text { risk of death in } \mathrm{A}}{\text { risk of death in } \mathrm{B}} .
$$

In a randomised trial, it is also the ratio of compared incidences:

$$
H R=\frac{(n b \text { deaths in } A / \text { total_followed })_{\text {arm A A }}}{(n b \text { deaths in B/total_followed })_{\text {arm B }}} .
$$

If $\mathrm{HR}>1$, the risk involved with $\mathrm{A}$ is greater than with $\mathrm{B}$, and we conclude that A has a harmful effect.

If $\mathrm{HR}=1$, the risk involved with $\mathrm{A}$ is equal to with $\mathrm{B}$, and we conclude that A has no effect.

If $\mathrm{HR}<1$, the risk involved with $\mathrm{A}$ is lower than with $\mathrm{B}$, and we conclude that $\mathrm{A}$ has a beneficial effect.

This measurement is a relative value contrasting with median survival, which is an absolute value, and therefore interpretation must take into account the comparator arm and rest on the hypothesis of risk proportionality. The significance of hazard ratios is that they measure the effect of treatment throughout follow-up.

For a given difference in median survival, the hazard ratio may reflect major deviations in the differentiation between two products and is therefore supplemental.

\subsection{Comparator}

Assessing the effect of treatment depends on the comparative nature of the trial and the relevance of the comparator treatment, which is judged by the trial population. The comparative trial may be versus a drug or recognised management strategy. If the chosen comparator is not recognised as one of the best by the Transparency Commission, there is a risk of decline in ASMR or even SMR. The difficulty in oncology of judging the relevance of the comparator lies in the rate of change in therapeutic strategy. The use of expert opinions is highly important in this context. For other aspects involved in choosing comparators, the Round Table proposes referring to the recommendations of Round Table 1: comparators (medicinal and non-medicinal) for MA, for public health, for payers and at European level.

\subsection{Target population}

The target population chosen for reimbursement is generally the MA population. However, in oncology as in other therapeutic fields, this may be limited to a subset of the MA population. In other cases, the Transparency Commission may define subpopulations with different levels of ASMR. Effect size in these subpopulations is assessed based on subgroup analyses, on the condition that these analyses are specified in the protocol. However, changes in scientific data, particularly the identification of biomarkers in the development process, should lead the Commission to consider efficacy data from post-hoc analyses, which were not initially specified in the protocol. In order to be admissible, 


\section{Accounting for therapeutic need in assessment of effect}

\section{Incidence and mortality of different cancers in 2005}

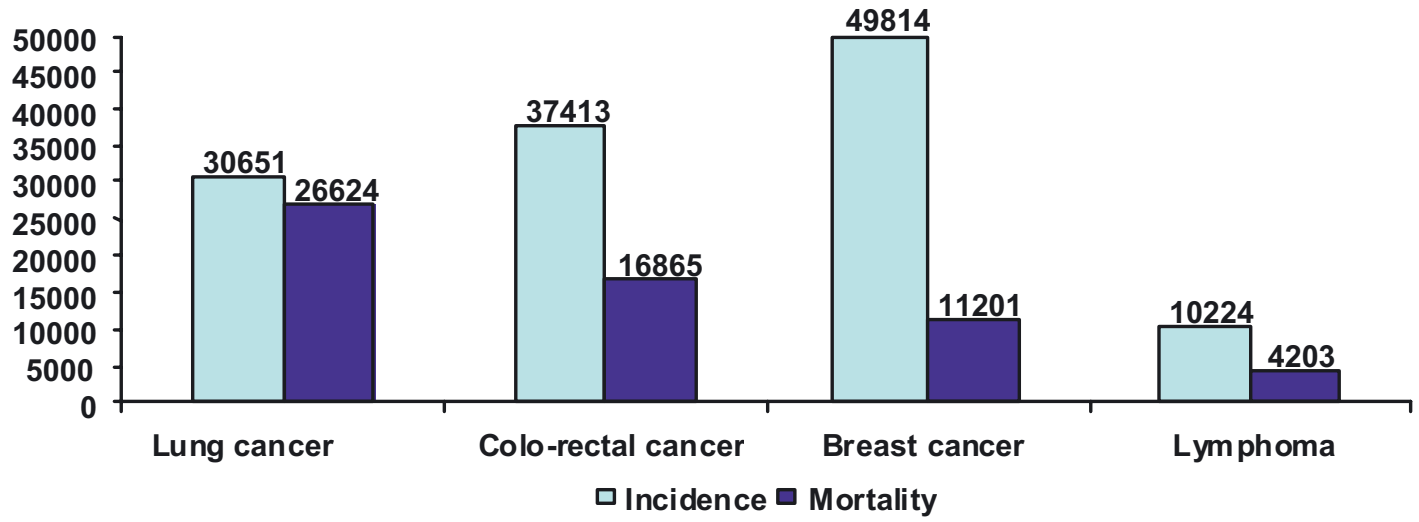

Fig. 1. Acounting for therapeutic need in assessment of effect.

these analyses must be performed with full methodological rigor, particularly an interaction test, and the Commission may, in order to validate the rigor, need to request the release of individual data. Another possibility suggested by the working group for targeted therapies for which data are not "mature" in a subpopulation, for example, is the granting of a temporary ASMR on the condition of confirmation by ad hoc assessment.

For other aspects of the target population, the roundtable proposes referring to the recommendations of Round Table 5: definition of target populations: how best to anticipate the definition of target populations for the purpose of reimbursement: data sources, reasoning, etc.

\subsection{Medical need}

Medical need in oncology cannot be addressed unequivocally since cancer covers a very diverse clinical and therapeutic reality. One way to address medical need is to compare the incidence and mortality of four common cancers (figure 1). Should a gain in survival of two months, i.e. a given absolute effect size, be assessed in the same manner in breast cancer as in lung cancer? Or should a given quantity of reduction in relative risk be assessed in the same manner in breast cancer as in lung cancer?

Translation of effect size into ASMR will take into account the baseline of the endpoint, and mean survival for the disease in question will be taken into account in order to assess added medical value. Therefore, the existence of medical need that is significant or not covered affects the assessment of treatments in general and cancer drugs in particular for reimbursement.

\section{Conclusion and recommendations}

In assessing cancer drugs for reimbursement, the Transparency Commission requested data on overall survival. In fact, overall survival (OS) is not always the ideal primary endpoint. Progression-Free Survival (PFS) for metastatic situations or Disease-Free Survival (DFS) in adjuvant situations are wholly relevant endpoints in slowly progressing diseases or when salvage treatments are available. However, in early lines of treatment, insofar as the ultimate therapeutic objective of a cancer drug is to prolong survival, if PFS is chosen as the primary endpoint, OS should still be taken as a secondary endpoint with monitoring of survival. Its interpretation may often raise questions, particularly when, for obvious ethical reasons, second-line treatments have been administered. As for response rate alone, it is not accepted as an endpoint in assessment for reimbursement by the Transparency Commission, except in malignant blood diseases.

Effect size is assessed using actuarial survival curves of the product versus the comparator. This information is often processed using median survival, but in the absence of an ideal parameter, the recommendation is to use several parameters: hazard ratio, median overall survival or progression-free survival and survival rate at a given time $t$.

Apart from the endpoints, assessing cancer drugs for reimbursement is no different from other therapeutic classes. The attempt by the working group to determine an effect size threshold for the granting of reimbursement did not succeed because it would depend on medical need, the comparator and progress in knowledge in the field in question. 
In fact, rapid development in reference therapeutic strategies, related firstly to the abundance of product portfolios in development (700 molecules in development) and secondly to the identification of predictive factors of efficacy (biomarkers or histology), has led the roundtable to make several recommendations. The first is the opportunity for pharmaceutical companies to hold informational meetings with early scientific opinions from the office of the Transparency Commission. The discussions would pertain mainly to the choice of comparators and relevance of responder subgroups, particularly the identification of new predictive response factors. This exchange may lead to the definition of 2 levels of ASMR in 2 subgroups of the MA population or in case of non significant clinical effect to the restriction of the target population to a subgroup in which the expected effect size is greater. The second recommendation is, in case of "immaturity" in certain efficacy results, the possibility of granting temporary transparency opinions, with a temporary ASMR, on the condition of confirmation.

Participants. Jean-Pierre Armand (Institut Claudius Regaud, Toulouse), Marc Bardou (CIC, Inserm, Dijon), Loïc Bergougnoux (Laboratoire Roche), Christine Bouchet (Laboratoire Novartis), Dominic Cellier (Laboratoire Merck), Bachir Dahmani (Haute Autorité de Santé, Saint-Denis), Bertrand Diquet (CHU, Angers), Thierry Lecomte (CHU, Tours), Nathalie Hoog Labouret (InCa, Boulogne Billancourt), Claire Labreveux (Laboratoire GlaxoSmithKline), François Meyer (Haute Autorité de Santé, SaintDenis), Gilles Paintaud (CHRU, Tours), Pascal Piedbois (Laboratoire Astra-Zeneca), Martine Pigeon (Cnamts, Paris), Olivier Amédée Manesme (Paris Biotech, Paris), Rémy Defrance (Laboratoire Pfizer), Dominique Tonelli (Laboratoire Bristol Myers Squibb), Karen Pinachyan (Laboratoire Murigenetics), Sophie Tardieu (AP-HM, Marseille)

\section{References}

1. EMEA. Guideline on the evaluation of anticancer medicinal products in man (CPMP/EWP/205/95 Rev.3/Corr.2, Dec 2005) http://www . ema . europa . eu/pdfs/human/ewp/020595en.pdf

2. FDA. Guidance for industry, clinical trial endpoints for the approval of cancer drugs and biologics (May 2007) http://www.fda.gov/downloads/ Drugs/GuidanceComplianceRegulatoryInformation/Guidances/ ucm071590.pdf
3. Mathoulin-Pelissier S, Gourgou-Bourgade S, Bonnetain F, et al. Survival end point reporting in randomized cancer clinical trials: a review of major journals. J Clin Oncol 2008; 26: 3721-6

4. Pazdur R. Endpoints for assessing durg activity in clinical trials. Oncologist 2008; 13(suppl2): 19-21

5. Pignatti F, Aronso B, Vamvakas S, et al. Clinical trials for registration in the European Union: the EMEA 5-year experience in oncology. Critical Reviews in Oncology/Hematology 2002; 42: 123-35

6. Eisenhauer A, Therasse P, Bogaerts $\mathrm{J}$, et al. New response evaluation criteria in solid tumours: revised REGIST guideline (version 1.1). European Journal of Cancer 2009; 45: 228-47

7. Cheson B, Pfistner B, Juweid ME, et al. Revised response criteria for malignat lymphoma. J Clin Oncol 25: 579-86

8. Buyse M, Burzykowski T, Caroll K, et al. Progression-free survual is a surrogate for survival in advanced colorectal cancer. J Clin Oncol 2007; 25: 5218-24

9. Punt CJ, Buyse M, Köhne $\mathrm{CH}$, et al. Endpoints in adjuvant treatment trials: a systematic review of the literature in colon cancer and proposed definitions for future trials. J Natl Cancer Inst 2007; 99: 998-1003

10. Sargent D, Wieand H, Haller D, et al. Disease-free survival Versus overall survival as a primary end point for adjuvant colon cancer studies: individual patient data from 20,898 Patients on 18 randomized Trials. J Clin Oncol 2005; 23: 8664-70

11. Tang P, Bentzen S, Chen E, et al. Surrogate end points for median overall survival in metastatic colorectal cancer: literature-based analysis from 39 randomized controlled trials of first-line chemotherapy. J Clin Oncol 2007; 25: $4562-8$

12. Hudis C, Barlow W, Costantino J, et al. Proposal for standardized definitions for efficacy end points in adjuvant breast cancer trials: The STEEP system. J Clin Oncol 2007; 25: 2127-32

13. Miksad R, Zietemann V, Gothe R, et al. Progression-free survival as a surrogate endpoint in advanced breast cancer. Int $\mathrm{J}$ of Technol Assess Health Care 2008; 24 (4): 371-83

14. Knox J. Progression-free survival as endpoint in metastatic RCC? Comment $\mathrm{n}^{\circ} 559157$ The Lancet Vol 372 August 9, 2008

15. Freidlin B, Korn E, Hunsberger S, et al. Proposal for the use of progressionfree survival in unblinded randomized trial. J Clin Oncol 2007; 25: 2122-6

16. Fleming $\mathrm{T}$, Rothman $\mathrm{M}$ and $\mathrm{Lu} \mathrm{H}$. Issues in using progression-free survival when evaluating oncology product. J Clin Oncol 2009; 27: 2874-80

17. Dodd L, Freidlin B, Jaffe C, et al. Blinded independent central review of progression-free srvival in Phase III clinical trials: important design element or unnecessary expense? J Clin Oncol 2008; 26: 3791-6

Correspondence and offprints: Rima de Sahb-Berkovitch, Affaires Économiques, Laboratoire Roche, 52 boulevard du Parc, 92521 Neuilly sur Seine Cedex, France.

E-mail: rima.de_sahb-berkovitch@roche.com 